\title{
Aplicação de cromatografia centrífuga de contra-corrente na purificação de ácido ursólico das folhas de Eugenia brasiliensis Lam.
}

\author{
N. Frighetto ${ }^{1 *}$, R.M.Welendorf ${ }^{1}$, A.M.P. Silva ${ }^{2}$, M.J. Nakamura ${ }^{2}$, A.C. Siani ${ }^{2}$ \\ ${ }^{I}$ Centro Pluridisciplinar de Pesquisas Quimicas, Biológicas e Agrícolas - CPQBA, Universidade Estadual de \\ Campinas - UNICAMP, C.P. 6171, Betel, 13083-970, Campinas, SP, Brasil, \\ ${ }^{2}$ Departamento de Química de Produtos Naturais, Instituto de Tecnologia em Fármacos, Fundação Oswaldo Cruz \\ FIOCRUZ, R. Sizenando Nabuco, 100, 21041-250, Rio de Janeiro, RJ, Brasil
}

\begin{abstract}
RESUMO: Os ácidos triterpênicos são metabólitos comuns na família Myrtaceae, especialmente no gênero Eugenia. O ácido ursólico foi descrito como um dos principais constituintes, nas folhas de Eugenia brasiliensis, coletada no Sudoeste do Brasil. Uma partição prévia, por solventes, do extrato etanólico ou do extrato clorofórmico de E. brasiliensis, seguida por uma purificação por cromatografia de contra-corrente de alta velocidade (CCCAV), conduziu ao isolamento do ácido ursólico com alto grau de pureza (>97\%). Esta substância, também foi isolada por cromatografia convencional de coluna aberta (rendimento de $0.22 \%$ a partir do extrato etanólico), e caracterizada por ${ }^{13} \mathrm{C}-\mathrm{RMN}, \mathrm{GC}-\mathrm{EM}$ e co-injeção com padrão comercial em CG-DIC, na forma do éster metílico. A técnica de CCCAV, usualmente usada para triterpenos glicosilados, foi aqui aplicada para a aglicona. As fases móvel e estacionária, no experimento de CCCAV, foram geradas pela mistura de n-hexano : acetato de etila : metanol : água, na proporção 10:5:2,5:1. A seleção do sistema de solventes (fases estacionária e móvel) foi determinada pela máxima distribuição eqüitativa do ácido ursólico em ambas as fases, medida por densitometria e monitorada por cromatografia em camada delgada, CCD, usando-se ácido ursólico comercial como referência.
\end{abstract}

Unitermos: Eugenia brasiliensis, Myrtaceae, acido ursólico, cromatografia de contra-corrente

\begin{abstract}
Aplication of counter-current chromatography in the purification of ursolic acid from leaves of Eugenia brasiliensis Lam.”. Triterpene acids are common metabolites in the Myrtaceae family, especially in the genus Eugenia. Ursolic acid was found in Eugenia brasiliensis collected in Southeastern Brazil. A previous solvent partition of the ethanol or chloroform extracts of the leaves of E. brasiliensis, followed by rapid high-speed counter-current chromatography (HSCCC) afforded ursolic acid in high purity (>97\%). This compound was also purified apart by conventional column chromatography (yield of $0.22 \%$ from the ethanolic extract) and characterized by ${ }^{13} \mathrm{C}-\mathrm{NMR}$, GC-MS and co-injection of its methyl ester with standards in GC-FID. The HSCCC technique, usually applied to triterpene glycosides, was here applied successfully to an aglycone, to which examples are rarely described. The mobile and stationary phase for the HSCCC experiment were derived from the two-phase solvent system composed by n-hexane : ethyl acetate : methanol : water in the proportion of 10:5:2.5:1. The choice of the developing solvent system for optimum HSCCC separation was determined by TLC coupled to densitometric measurements of ursolic acid in both stationary and mobile phase, generated by the upper and lower layer of the system above. Commercial ursolic acid was used as standard.
\end{abstract}

Keywords: Eugenia brasiliensis, Myrtaceae, ursolic acid, counter-current chromatography.

\section{INTRODUÇÃO}

Uma grande diversidade de vegetais, normalmente utilizados como alimentos ou com aplicação medicinal, apresenta ácidos triterpênicos em sua constituição química, tanto na forma livre, quanto na forma de agliconas de saponinas triterpenóides. Na família Myrtaceae, em especial em várias espécies do representativo gênero Eugenia, relata-se a presença de ácidos triterpênicos em várias espécies. De acordo com a espécie da qual foram isoladas, as estruturas destes metabólitos usualmente variam entre os esqueletos ursano, oleonano e lupano, contendo normalmente um ou dois grupos hidroxilas. Esta correlação pode ser visualizada na Tabela 1.

Os ácidos triterpênicos mono-hidroxilados (oleanólico, ursólico e betulínico) foram muito investigados no tocante ao seu amplo espectro de atividades biológicas, onde se destacam as atividades: antiinflamatória (Liu, 1995), antineoplásica (Tokuda et al., 1986; Pisha et al., 1995), antivirótica (Pavlova et al., 2003), antimicrobiana (Sattar et al., 1995), antiparasitária (Leite et al., 2001), hepatoprotetora (Saraswat et al., 1996), e outras. Estes ácidos apresentam uma baixa ou nenhuma toxicidade, sendo, inclusive, utilizados como aditivos em bebidas, alimentos e em cosméticos (Leung; 


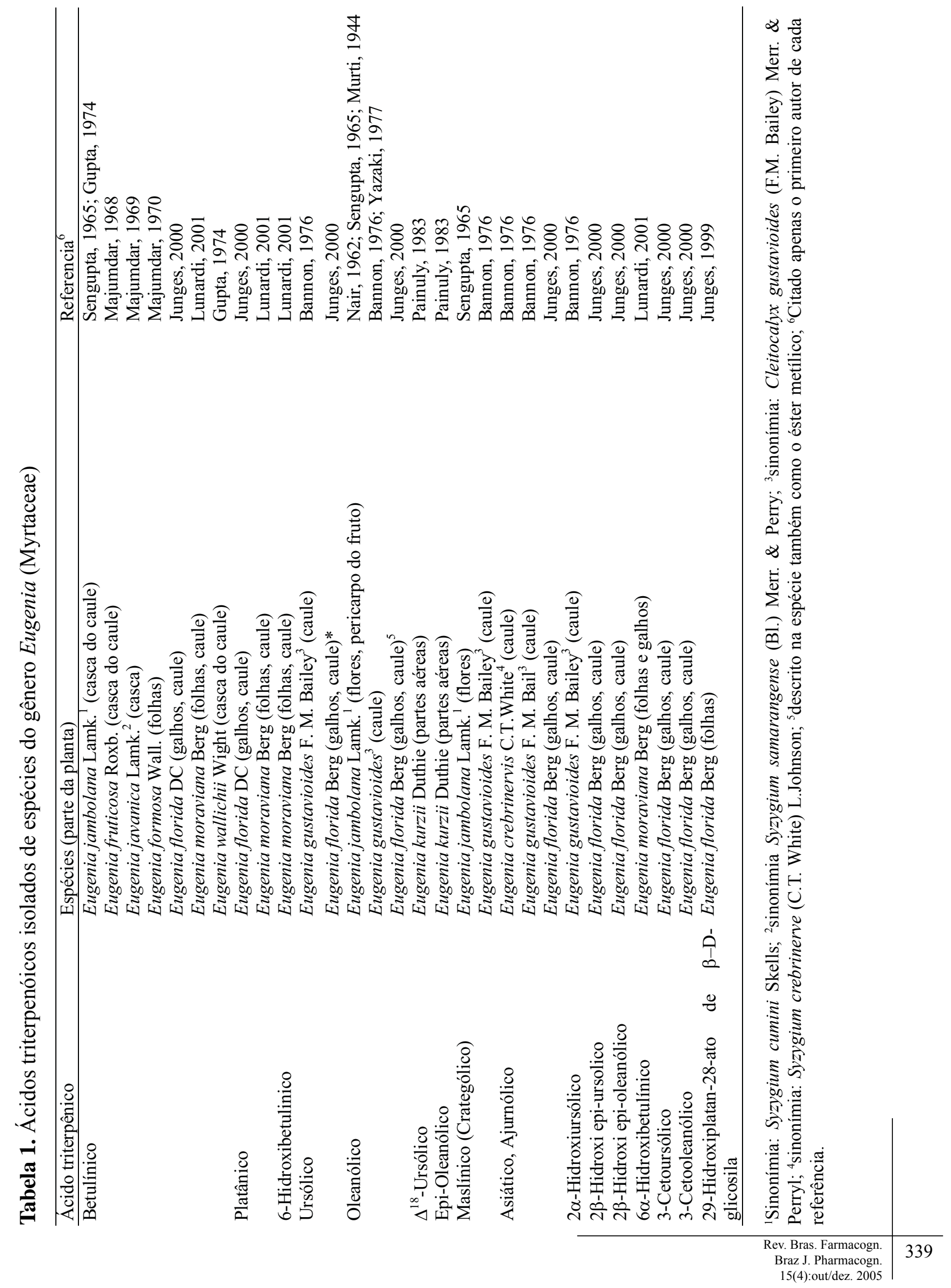


Foster, 1996). A disponibilização de ácidos triterpênicos para a composição de medicamentos ou outras aplicações exige a purificação destas substâncias, a partir de fontes naturais, em processos que sejam industrialmente viáveis e, portanto, adaptados à escala de produção. Apesar do ácido betulínico estar presente em inúmeras espécies tropicais (Hayek et al., 1989); as fontes tradicionais dessa substância são espécies européias do gênero Betula, que produzem o álcool precursor, a betulina (Kim et al., 1997). Recentemente, a técnica de cromatografia de contracorrrente de alta velocidade (CCCAV) foi descrita como uma alternativa para a purificação de ácido betulínico a partir de extratos brutos ou semipurificados de Eugenia florida (Frighetto et al., 2002).

O presente trabalho reporta o isolamento e a purificação por cromatografia de contra-corrente de alta velocidade (CCCAV), de um outro ácido triterpênico de aplicação medicinal, o ácido ursólico, a partir de extratos brutose semi-purificados das folhas de Eugenia brasiliensis Lam., espécie de Myrtaceae descrita como anti-reumática conhecida popularmente como "grumixama" (Corrêa, 1984). Nesta técnica cromatográfica, a composição do sistema bifásico de solventes a ser utilizado como fase estacionária e como fase móvel foi, primeiramente, estabelecido através de cromatografia em camada delgada (CCD), buscando-se condições otimizadas para uma boa resolução do material a ser separado e purificado na coluna (bobina). Além disso, como um dos critérios de eficiência do sistema bifásico, as fases resultantes devem ser susceptíveis de uma imediata separação e recomposição, após agitação intensa (Conway, 1990). O processo requer ainda que a amostra a ser analisada apresente boa solubilidade no sistema bifásico de solventes e, fundamentalmente, alcance uma distribuição eqüitativa, o mais próximo possível da unidade (1:1) em ambas as fases (Hostettmann; Marston, 2001). Para atingir estes objetivos, as concentrações de ácido ursólico, em ambas as fases testadas, foram monitoradas por CCD, cuja placa de sílica gel foi avaliada mediante leitura ótica, por meio de densitômetro. Este procedimento parametrizou a seleção da composição do sistema quaternário-bifásico de solventes para gerar as fases estacionária e móvel a serem utilizadas no CCCAV. Vários parâmetros operacionais foram ensaiados, como a escolha da fase estacionária e da fase móvel, o sentido do fluxo do solvente dentro da coluna, se na direção "cabeça-cauda" ou "cauda-cabeça", a orientação de rotação da coluna, no sentido horário ou anti-horário, a velocidade de rotação desta, expressa em rpm; os volumes relativos das fases móvel e estacionária, e a concentração da amostra injetada.

\section{MATERIAL E MÉTODOS}

\section{Material vegetal}

As folhas de Eugenia brasiliensis Lam. foram coletadas nas cercanias do campus da Universidade
Estadual de Campinas, em Agosto de 1996. A espécie foi identificada pela Dra. Graziela M. Barrozo (in memoriam), e uma exsicata foi preparada e depositada no Herbário do Jardim Botânico do Rio de Janeiro, sob código RB \#326234.

\section{Obtenção dos extratos}

As folhas $(1,412 \mathrm{Kg})$, após secagem à temperatura ambiente, foram trituradas em um moinho de facas e, em seguida, maceradas com etanol absoluto $(10 \mathrm{~L})$, durante 8 dias. A filtração e evaporação do solvente forneceram 134,2 g $(9.50 \%)$ de extrato I. A re-extração do resíduo de folhas com uma segunda carga de etanol (10 L, 8 dias) resultou em 58,3 g (4.13\%) de extrato II. As amostras I e II foram submetidas à cromatografia líquido-líquido, no CCCAV.

\section{Isolamento do ácido ursólico}

A $20.4 \mathrm{~g}$ do extrato I juntou-se $\mathrm{CHCl}_{3}(2 \times 500$ $\mathrm{mL})$ e a fração solúvel $(2.35 \mathrm{~g})$, após evaporação, foi purificada por tratamento com éter de petróleo $(3 \times 100$ $\mathrm{mL})$. A parte insolúvel $(0.400 \mathrm{~g})$ foi cromatografada em coluna de sílica gel G 60, utilizando-se, como eluente, uma mistura gradiente de $\mathrm{MeOH}$ em $\mathrm{CHCl}_{3}(0,1,3$, $5,8,10,13,15,20$ e $100 \%$ de metanol; $50 \mathrm{~mL}$ cada). Foram coletadas 50 frações de $10 \mathrm{~mL}$, posteriormente reunidas segundo similaridade em $\mathrm{CCD}$, utilizandose como eluente $\mathrm{CHCl}_{3}: \mathrm{MeOH}$ 9:1. As frações 24 a 27 foram agrupadas, evaporadas, re-dissolvidas em $\mathrm{CH}_{2} \mathrm{Cl}_{2} \mathrm{e}$ purificadas com carvão ativado (agitação, $2 \mathrm{~h}$ ), fornecendo 45,3 mg de ácido ursólico.

\section{Instrumentação e material cromatrográfico}

O espectro de IV foi obtido em espectrofotômetro Nicolet 205. O espectro de ${ }^{13} \mathrm{C}$-RMN foi obtido em um espectrômetro Bruker AC 200-A (50,3 MHz), usandose $\mathrm{CDCl}_{3}$, como solvente. A análise por CG-EM foi realizada em um equipamento HP, Mod. 6890, acoplado a um detector de massas, HP Mod. 5971, dotado de biblioteca de espectros Wiley (software 59943B). Foi utilizada uma coluna capilar MS-HP-5 (30m x 0,32 mm $\mathrm{x} 0,25 \mu \mathrm{m}$ de filme), gás carreador $\mathrm{He}$ (fluxo $0,5 \mathrm{ml} / \mathrm{min}$ ), T-injetor $250^{\circ} \mathrm{C}$, e taxa de split $1 / 20$. As condições de operação foram: Ti $150{ }^{\circ} \mathrm{C}$, ti 0 min, Tf $280{ }^{\circ} \mathrm{C}$, taxa de aquecimento $10 \% \mathrm{~min}$, fluxo de $\mathrm{He}: 1 \mathrm{ml} / \mathrm{min}$; injeção de $1 \mu \mathrm{L}$ de uma solução de 4-6 mg de ácido ursólico em 1 $\mathrm{mL}$ de $\mathrm{CH}_{2} \mathrm{Cl}_{2}$.

Nas medidas de densitometria ótica, utilizouse um densitômetro Pharmacia-Biotech, acoplado ao software apropriado. Os experimentos de CCCAV foram executados em um aparelho P. C. (Potomac, USA), equipado com coluna múltipla, para análises em diferentes escalas. 


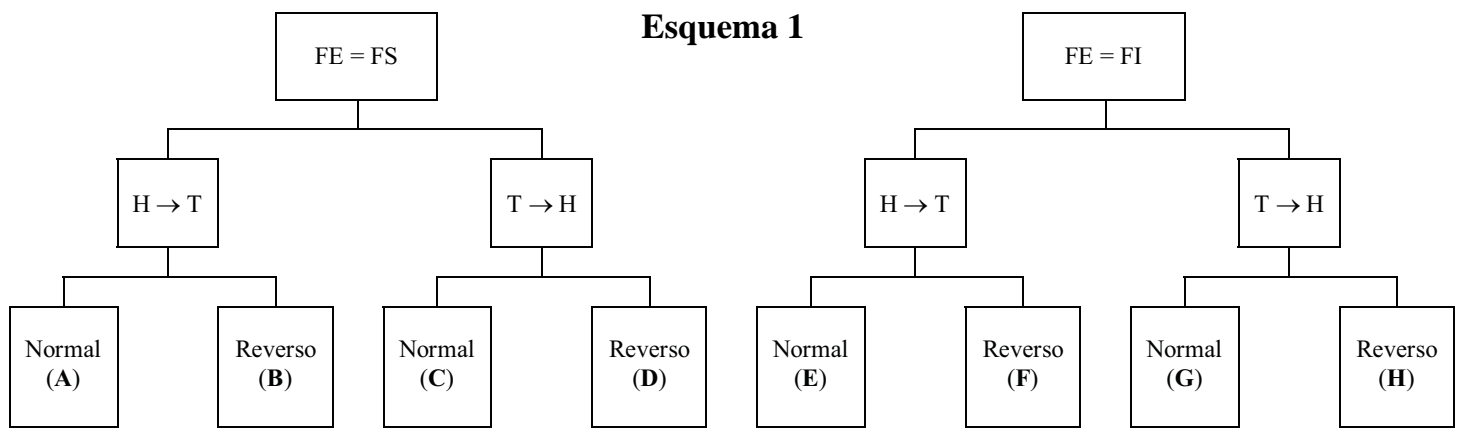

$\mathrm{FE}=$ Fase estacionária; $(\mathrm{H}) \rightarrow(\mathrm{T})=$ Fluxo do sistema de solventes no sentido: Cabeça (head) $\rightarrow$ Cauda (tail); $(\mathrm{T}) \rightarrow(\mathrm{H})=$ Fluxo do sistema de solventes no sentido: Cauda (tail) $\rightarrow$ Cabeça (head). Normal e Reverso referem-se ao sentido de rotação da bobina no sentido horário ou anti-horário, respectivamente. Para o sistema de solventes escolhido (n-hexano:AcOEt:MeOH: $\mathrm{H}_{2} \mathrm{O}$ 10:5:2,5:1) e testes realizados a 960 rpm: Caminhos dos fluxogramas: (A) - Retém a fase estacionaria com sangria de 3,6\%; (B) - Não retém a fase estacionária; (C) - Não retém a fase estacionária; (D) - Retém a fase estacionaria com sangria de 7,2\%; (E) - Não retém a fase estacionária; (F) - Retém a fase estacionaria com sangria de 23,2\%; (G) - Retém a fase estacionaria com sangria de 14,5\%; (H) - Não retém a fase estacionária.

\section{Identificação do ácido ursólico}

Após metilação com diazometano, o ácido ursólico foi identificado por IV, ${ }^{13} \mathrm{C}-\mathrm{RMN}$, CG-EM (fragmentação característica e co-injeção com padrão de ácido ursólico comercial metilado) (Budzikiewicz et al., 1963) e CCD (comparação com padrão em diferentes sistemas de solventes). O ácido ursólico livre forneceu um espectro de IV totalmente superponível ao do padrão comercial $\left(\mathrm{C}=\mathrm{O}, \eta_{\max } 1693 \mathrm{~cm}^{-1}\right)$. O padrão de fragmentação obtido para o ursolato de metila (tempo de retenção 26.32 min. nas condições utilizadas) foi comparado com os espectros de massas do banco de dados Wiley, demonstrando compatibilidade acima de $97 \%[\mathrm{~m} /$ z $\left.470\left(\mathrm{M}^{+}\right), 455,410,262(100 \%), 203,189\right]$. Os sinais característicos em ${ }^{13} \mathrm{C}-\mathrm{RMN}$, para o ursolato de metila, utilizados como diagnóstico da estrutura do triterpeno pentacíclico, foram $(\delta$, ppm): 79,7 $(\mathrm{C} 3 ; \mathrm{COH}), 126,3$ (C12), $138,9(\mathrm{C}-13)$ e $178,8(\mathrm{C}-28 ; \mathrm{C}=\mathrm{O})$. As comparações do ácido ursólico isolado com o padrão comercial foram realizadas em CCD (sílica gel GF 60), utilizando-se como eluentes as misturas de $\mathrm{CHCl}_{3}: \mathrm{MeOH}: \mathrm{Et}_{2} \mathrm{O}$ 90:5:5 e benzeno:acetona 75:2; sistemas onde o ácido ursólico possui $R_{\mathrm{f}}$ de 0,49 e 0,52 , respectivamente. As placas foram reveladas com iodo ou solução de anisaldeído ou vanilina em $\mathrm{H}_{2} \mathrm{SO}_{4}$.

Seleção do sistema de solventes e doseamento do ácido ursólico via densitometria ótica

Diferentes composições de um sistema quaternário, constituído de uma mistura de n-hexano $(12.5 \%$ a $25 \%)$, acetato de etila ( $12.5 \%$ a $25 \%)$, metanol $(6.25 \%$ a $25 \%)$ e água $(1.25 \%$ a $25 \%)$ foram ensaiados no experimento de CCD. As amostras foram preparadas, diluindo-se os extratos I e II (2 a $5 \mathrm{mg}$ ) na mistura de solventes em diferentes proporções ( $2 \mathrm{~mL}$ de cada fase), e aplicadas separadamente em placas de sílica gel GF 60 (200 $\mathrm{mm} \times 100 \mathrm{~mm} \times 0,2 \mathrm{~mm}$ ), usando-se uma pipeta de $10 \mu \mathrm{L}$. Foram também aplicadas, na mesma placa, alíquotas entre 0.50 e $2.0 \mu \mathrm{g} / \mathrm{mL}$ de solução de ácido ursólico padrão $(2 \mathrm{mg} / \mathrm{mL})$, com o objetivo de se atingir diferentes concentrações nos spots. A placa foi então eluída em hexano:AcOEt (1:1). Após a eluição completa, os sinais pertinentes ao ácido ursólico foram revelados com anisaldeído/ácido acético/ácido sulfúrico em álcool etílico (Wagner; Bladt, 1996) e, então, submetidos às medidas de densitometria ótica. As medidas no densitômetro foram computadas pelo número de pixels e as quantidades de ácido ursólico, presentes em cada fase, calculadas por comparação com as respostas fornecidas pelos sinais do padrão, parametrizados numa curva: concentração vs. $\mathrm{n}^{\mathrm{o}}$ de pixels. Todos os experimentos foram efetuados em duplicatas e a média das medidas consideradas.

\section{Condições experimentais nos ensaios CCCAV}

A escala analítica (volume da coluna de 14,8 $\mathrm{mL}$ ) do equipamento de CCCAV permitiu injeções de até $20 \mathrm{mg}$ de amostra (extratos I e II). As análises semipreparativas (coluna de $80 \mathrm{~mL}$ ) e preparativas (coluna de $230 \mathrm{~mL}$ ) permitiram injeções de $100 \mathrm{mg}$ até $500 \mathrm{mg}$ de amostra, respectivamente, utilizandose um sistema quaternário-bifásico de solventes, nas condições de otimização descritas anteriormente e uma velocidade roto-elicoidal fixa da coluna (960 rpm). Uma série de ensaios foi realizada com o objetivo de definir as melhores condições operacionais para os experimentos. Para tanto, oito ensaios foram realizados, alternando cada uma das fases, como fase estacionária ou fase móvel, objetivando maximizar a retenção da fase estacionária, 
no sentido de minimizar sua co-eluição com a fase móvel através da coluna; variando-se a combinação de diferentes parâmetros, como: (i) bombeamento do fluxo de solventes nos sentidos cabeça-cauda x cauda-cabeça; (ii) rotação da bobina no sentido normal (horário) e sentido reverso (anti-horário), conforme está explicitado no Esquema 1.

Com base na otimização dos ensaios anteriores, todos os experimentos foram realizados com fluxo de 1,0 $\mathrm{mL} / \mathrm{min}, 4,0 \mathrm{~mL} / \mathrm{min}$ e de 2,0 a $4,0 \mathrm{~mL} / \mathrm{min}$ nas colunas analítica, semipreparativa e preparativa, respectivamente. Um ensaio foi realizado com uma coluna com capacidade de $325 \mathrm{~mL}$, obtida pela interligação das três colunas, que suportou carga de $1000 \mathrm{mg}$ de amostra para uma única análise. O volume de fase móvel utilizado foi sempre o dobro do volume da fase estacionária em cada experimento. Foram coletadas frações entre 3-4 $\mathrm{mL}$, as quais foram monitoradas por CCD e reunidas posteriormente, conforme a similaridade na composição. Ao final de cada experimento, a fase estacionária foi exaurida completamente da coluna e concentrada em evaporador rotatório, assegurando-se, através de pesagem, a recuperação da totalidade de amostra introduzida na coluna.

\section{RESULTADOS E DISCUSSÃO}

Os experimentos de $\mathrm{CCD} /$ densitometria definiram o melhor sistema de solventes como a mistura composta por $n$-hexano : acetato de etila : metanol : água, nas proporções 10:5:2.5:1, que resultou numa relação muito próxima à distribuição eqüitativa $(1: 1,15)$ das concentrações de ácido ursólico na fase superior e inferior, respectivamente. Conforme está evidenciado no Esquema 1, apenas duas, entre as oito possibilidades, foram adequadas, no sentido de manter a fase estacionária em um nível aceitável de perda por arraste da coluna $(96,4 \%$ a $76,8 \%$ ), segundo os caminhos $\mathbf{A}$ e $\mathbf{D}$, respectivamente. A condição $\mathbf{D}$ foi eleita como a mais eficiente, uma vez que A, embora com menor volume de co-eluição, não conduziu a uma boa separação do ácido ursólico, provavelmente, devido à interferência de outros constituintes presentes nos extratos. Conforme mostrado no ensaio D, a fase superior do sistema de solventes foi utilizada como a fase móvel, levando à obtenção do ácido ursólico com alto grau de pureza, atestada pelo único sinal em $\mathrm{CG}$, pelo espectro de ${ }^{13} \mathrm{C}-\mathrm{RMN}$ e por comparação cromatográfica com a substância isolada por cromatografia em coluna aberta. Os rendimentos de ácido ursólico variaram entre $87-96 \%$ para os experimentos realizados nas escalas preparativa, semi-preparativa e analítica, com os extratos I e II.

Estes resultados credenciam o uso da técnica de densitometria acoplada a CCD, como uma ferramenta útil para o sucesso dos experimentos de separação e purificação em CCCAV, de ácidos triterpênicos, a partir de extratos vegetais brutos ou semipurificados.

A técnica de CCCAV, usualmente aplicada para a purificação de muitas classes de substâncias é, freqüentemente, descrita para saponinas de esteróides e triterpenos, flavonóides, alcalóides e outros (Marston; Hostettmann, 1994). Contudo até o momento, foi encontrada apenas uma citação da aplicação desta técnica para ácidos triterpênicos (Ito, 1990), mesmo assim, utilizando misturas menos complexas de substâncias do que aquelas presentes nos extratos das folhas, como apresentado neste trabalho. Neste sentido, as espécies contidas na Tabela 1 poderiam ser exploradas para a obtenção desse grupo de metabólitos, utilizando-se inicialmente as condições aqui desenvolvidas. Além do extrato alcoólico, outros solventes de diferentes polaridades devem ser ainda testados, no intuito de extrair os ácidos triterpênicos a partir de folhas de diferentes mirtáceas. Solventes que reduzam a extração de clorofila nos extratos a serem purificados por CCCAV seriam preferíveis, mas esta seleção está condicionada à conformação do esqueleto, assim como o grau de hidroxilação dos ácidos triterpênicos presentes no vegetal.

Por outro lado, a técnica de CCCAV deveria ser mais bem aproveitada, tanto na separação, quanto na purificação de constituintes vegetais, visando à obtenção de substâncias de referência e de padrões, principalmente quando se almeja baixar custos operacionais, por intermédio da diminuição do tempo de análise e da utilização de solventes sem alto grau de pureza, como aqueles utilizados em CCCAV. Além disso, esta técnica permite a recuperação de todo o material vegetal sob análise, evitando perdas pela adsorção ou outros tipos de fatores, inerentes à cromatografia convencional.

\section{REFERÊNCIAS}

Bannon CD, Eade RA, Simes JJH 1976. Extractives of Australian Timbers. XVI. The Constituents of the wood of Eugenia crebrinervis (syn. Syzigium crebrinervis) and Eugenia gustavioides (syn. Cleitocalyx gustavioides). Aust J Chem 29: 1135-1141.

BudzikiewiczH, Wilson JM, Djerassi C 1963. Mass spectrometry in structural and stereochemical problems. XXXII. Pentacyclic triterpenes. J Am Chem Soc 85: 36883699.

Conway WD 1990. Countercurrent chromatography: Apparatus, theory and applications. New York: VCH Publishers Inc., J. Wiley \& Sons.

Corrêa P 1984. Dicionário das plantas úteis do Brasil e das exóticas cultivada. Vol IV. Rio de Janeiro: Imprensa Nacional, Ministério da Agricultura, p. 429-430.

Frighetto N, Terci D, Welendorf RM, Siani AC 2002. Aplicação da técnica HSCCC no isolamento do ácido betulínico a partir do extrato etanólico de Eugenia florida DC (Myrtaceae). XVII Simpósio de Plantas Medicinais do Brasil, Cuiabá, Brasil.

Gupta GS, Sharma DP 1974. Triterpenoid and other constituents of Eugenia jambolana leaves. Phytochemistry 13: 2013-2014

Hayek EWH, Jordis U, Moche W, Sauter F 1989. A bicentennial 
of betulin. Phytochemistry 28: 2229-2242.

Hostettmann K, Marston A 2001. Countercurrent chromatography in the preparative separation of plant-derived natural products. J Liq Chromatogr Relat Technol 24: 1711-1721.

Ito Y, Oka H, Kitazume E, Bhatnagar M, Lee YW 1990. Improved high-speed countercurrent chromatograph with three multilayer coils connected in series. III. Evaluation of semianalytical column with various biological samples and inorganic elements. $J$ Liq Chromatogr 3: 2329-2349.

Junges MJ, Fernandes JB, Vieira PC, Silva MFGF, RodriguesFilho E 1999. The use of C-13 and H-1-NMR in the structural elucidation of a new nor-lupane triterpene. J Braz Chem Soc 10: 317-320.

Junges MJ, Fernandes JB, Vieira PC, Fernandes MFGS, Rodrigues-Filho E, Frühauf M, Barañano AG 2000. Triterpenos ursânicos e oleanânicos isolados do caule de Eugenia florida DC. Revista de Pesquisa e Pós-graduação da Universidade Regional Integrada do Alto Uruguai e das Missões 1: 13-29.

Kim DSHL, Chen Z, Nguyen VT, Pezzuto JM 1997. A concise semi-synthetic approach to betulinic acid from betulin. Synth Commun 29: 1607-1612.

Leite JPV, Lombardi JA, Chiari E, Oliveira AB 2001. Isolamento biomonitorado de uma substância tripanossomicida de Arrabidaea triplinervia (Bignoniaceae), o ácido ursólico. Rev Bras Farmacogn 11: 77-87.

Leung AY, Foster S 1996 Encyclopedia of common natural ingredients used in food, drug and cosmetics. $2^{\text {nd }}$ ed. New York: John Wiley \& Sons Inc.

Liu J 1995. Pharmacology of oleanolic acid and ursolic acid. $J$ Ethnopharmacol 49: 57-68.

Lunardi I, Peixoto JLB, Silva CC, Shuquel TA, Basso EA, Vidotti GJ 2001. Triterpenic acids from Eugenia moraviana. J Braz Chem Soc 12: 180-183.

Majumdar SG, Thakur S 1968. Chemical investigation of the stem-bark of Eugenia fruticosa Roxb and bark of Eugenia wallichii Wight III. J Ind Chem Soc 45: 785-788.

Majumdar SG, Thakur S 1969. Chemical investigation of the bark of Eugenia javanica Lamk. J Ind Chem Soc 46: 785-788.

Majumdar SG, Thakur S 1970. Chemical investigation of the bark of Eugenia formosa Wall. J Ind Chem Soc 47: 181-182.

Marston A, Hostettmann K 1994. Counter-current chromatography as a preparative tool - applications and perspectives. J Chromatogr A 658: 315-341.

Murti PB, Rao NVS 1944. Wax and resin components of the pericarp of jambul fruits (Eugenia jambolana). Proc Ind Acad Sci 20A: 163-168.

Nair AGR, Subramanian S 1962. Chemical examination of flowers of Eugenia jambolana. $J$ Sci Ind Res India 21B: 457-458.

Painuly P, Tandon JS 1983. A new triterpene from Eugenia kurzii Duthie. Indian J Chem 22B: 284-285.

Pavlova NI, Savoniva OV, Nikolaeva SN, Boreko EI, Flekhter OB 2003. Antiviral activity of betulin, betulinic and betulonic acids against enveloped and nonenveloped viruses. Fitoterapia 74: 489-492.

Pisha E, Chai H, Lee IS, Chagwedera TE, Farnsworth NR, Cordell GA, Beecher CWW, Fong HHS, Kinghorn
AD, Brown DM, Wani MC, Wall ME, Hieken TJ, Gupta TKD, Pezzuto JM 1995. Discovery of betulinic acid as a selective inhibitor of human melanoma that functions by induction of apoptosis. Nat Med 1: 1046-1051.

Saraswat B, Visen PKS, Dayal R, Agarwal DP, Patnaik GK 1996. Protective action of ursolic acid against chemical induced hepato-toxicity in rats. Indian $J$ Pharmacol 28: 232-239.

Sattar A, Bamkova V, Kujumgiev A, Galabov A, Ignatova A, Todorova C, Popov S 1995. Chemical composition and biological activity of leaf exudates from some Lamiaceae plants. Pharmazie 50: 62-65.

Sengupta P, Bikram P 1965. Triterpenoids and related compounds. IV. Triterpenoids from the stem-bark of Eugenia jambolana. J Ind Chem Soc 42: 255-258, apud Chemical Abstracts 63: 7053e.

Sengupta P, Bikram P 1965. Triterpenoids from the flowers of Eugenia jambolana. J Ind Chem Soc 42: 539-542.

Tokuda H, Ohigashi H, Koshimizu K, Ito I 1986. Inhibitory effects of ursolic and oleanolic acid on skin tumor promotion by 12-O-tetradecanoylphorbol-13acetate. Cancer Lett 33: 279-285.

Yazaki Y 1977. Polyphenols and triterpenes of Eugenia gustavioides wood. Phytochemistry 16: 138-139.

Wagner H, Bladt S 1996. Plant drug analysis. 2 ed., Berlin Heidelberg: Springer. 